\title{
COPY NUMBER CHANGES IN 1q21.3 AND 1q23.3 HAVE DIFFERENT CLINICAL RELEVANCE IN OVARIAN TUMORS
}

\author{
Dimova I ${ }^{1}$, Orsetti B ${ }^{2}$, Theillet $\mathrm{Ch}^{2}$, Dimitrov R. ${ }^{3}$, Toncheva DI ${ }^{1, *}$
}

*Corresponding Author: Draga I. Toncheva, Department of Medical Genetics, Medical Faculty, Medical University, 2 Zdrave str. SBALAG "Maichin dom," 6 Fl., 1431, Sofia, Bulgaria; Tel./Fax: +35-92-952-0357; E-mail: dragatoncheva@yahoo.com

\begin{abstract}
Many studies have reported aberrations such as amplifications, deletions and translocations of 1q21q23 in ovarian tumors. These findings increase the scientific interest in analyzing this region using specific gene probes. We investigated the frequency of copy number changes of two specific bacterial artificial chromosomes (BAC) clones in 1q21.3 and 1q23.3 by fluorescent in situ hybridization (FISH) on tissue microarrays consisting of 540 ovarian tumors of different malignancies, histology, stage and grade. Such changes in 1q21.3 were established in $9.64 \%$ of malignant ( $2.41 \%$ amplification), in $8.33 \%$ of low malignant potential (LMP) and in 13.13\% of benign ovarian tumors. Copy number changes of 1q23.3 were found in $17.78 \%$ of malignant $(1.48 \%$ amplification), in $16.67 \%$ of LMP and in $12.64 \%$ of benign ovarian tumors. We found a significantly higher gain of 1q23.3 in non epithelial $(50 \%)$ compared to epithelial tumors $(14.73 \%)(p<0.03)$. The gain of 1q21.3 prevailed in non serous malignant and LMP ovarian tumors in comparison to serous tumors. In non serous tumors, both gains were associated with higher grade. The frequency of gain
\end{abstract}

1 Department of Medical Genetics, Medical University of Sofia, Sofia, Bulgaria

2 Laboratory "Identity and Plasticity of Tumors", INSERM, Montpellier, France

3 Second Gynecologic Clinic, University Hospital of Obstetrics and Gynecology "Maichin dom", Sofia, Bulgaria in $1 \mathrm{q} 23.3$ was 2.5 -times higher than that in $1 \mathrm{q} 21.3$ of ovarian cancers.

Key words: Ovarian cancer, Copy number changes, Bacterial artificial chromosomes (BAC) clones, Fluorescent in situ hybridization (FISH), Tissue microarray (TMA)

\section{INTRODUCTION}

Cytogenetic studies and spectral karyotyping have shown chromosome 1 to be frequently involved in rearrangements in ovarian tumors [1-5]. However, specific genes in chromosome 1, of importance for ovarian carcinogenesis, are still not identified. To elucidate the mechanism of carcinogenesis, detailed analysis of gene amplifications and deletions in different cancers is needed. Comparative genomic hybridization (CGH) was introduced in 1992 for identifying DNA copy number changes in different types of tumors with a resolution, higher than that of chromosomal analysis, but of not more than 5-10 $\mathrm{Mb}$ [6]. The CGH studies showed its efficiency for discovering the regions involved in carcinogenesis, and eventually for determining their diagnostic and prognostic significance [7]. The CGH analysis of ovarian tumors resistant to standard platinum-based chemotherapy revealed that genetic gain of 1q21q22 is the most common alteration present [8]. This showed that there are different genetic changes in different groups of tumors and transfer efforts of investigation to analysis of alterations in chromosome 1, which may be of importance for the clinical course of the disease. 
Consistently higher transcription activity in a new large region of the long arm of chromosome 1 [1q21-q23 (140-160 Mb)] was established in many malignant, including ovarian tumors. For identification of the most common amplified loci of 1q in ovarian tumors, real time polymerase chain reaction (PCR) of 10 gene loci on this arm showed that MUCl was the most frequently amplified of them and significantly more often in platinum-resistant ovarian tumors (92\%) compared to well responsive tumors (21\%) [9]. Aberrations involving the region 1q21 have been found in ovarian tumors [10]. These findings increased the scientific interest in analysis of this region using specific gene probes. We investigated the frequency of copy number changes of two specific BAC (bacterial artificial chromosomes) clones in 1q21.3 and 1q23.3 in a large number of ovarian tumors of different malignancy, histology, stage and grade, and correlated these with tumor phenotype.

\section{MATERIALS AND METHODS}

Ovarian Tumors for Tissue Microarray (TMA) Construction. A TMA containing 540 ovarian tumor samples from 540 patients was constructed. The average age of the patients in the cohort was 55.25 years. There were 278 malignant tumors, 39 low malignant potential tumors (LMP) and 223 benign tumors (Table 1). The histological variants were: 248 serous, 61 mucinous, 85 endometrioid, two clear cell tumors, two Brenner tumors, 20 mixed epithelial tumors, 31 undifferentiated, 21 unclassified and 50 non epithelial tumors (granulosacell, germline-cell). The slides from all tumors were previously reviewed by a single pathologist. Tumor stage and grade were defined according to the International Federation of Gynecology and Obstetrics (FIGO) and World Health Organization (WHO) criteria [11].

Investigated Bacterial Artificial Chromosome Clones from 1q and Genes They Contain. We investigated the BAC of 1q21.3-RP11-126K1, of 168,686 bp (http://genome.ucsc.edu/). This contains the following genes: PIP5K1A; RFX5; PIK4CB; SELENBP1, which encode for phosphaditylinositol-4-phosphate-5-kinase $\alpha$, RFX5 protein (involved in expression of MHC-II), phosphaditylinositol-3-kinase, and Selen-binding protein 1, respectively.

We also investigated the BAC of 1q23.3RP11-97G24, of $171,855 \mathrm{bp}$. It contains the genes SLAMF1; SLAMF6; CD84, which encode for signaling lymphocytic activation molecule family member 1 (involved in cytolysis activity of NK cells), signaling lymphocytic activation molecule family member 6 , and protein of super family of immunoglobulin receptors, respectively.

Tissue Microarray. For TMA construction, a hematoxilin and eosin (H\&E)-stained section was made from each block to define representative tumor areas. Tissue cylinders with a diameter of $0.6 \mathrm{~mm}$ were punched from tumor areas and brought into a recipient paraffin block using a custom-made precision instrument [12]. Samples were distributed in

Table 1. Copy number changes of 1q21.3 in different stage tumors

\begin{tabular}{|l|l|c|c|c|c|}
\hline \multicolumn{2}{|c|}{} & Normal Copy Number (\%) & Genetic Gain (\%) & Amplification (\%) & Total (\%) \\
\hline \multirow{3}{*}{ Stage I } & Serous & $33(86.84)$ & $4(10.53)(p=0.28)$ & $1(2.63)(p=0.79)$ & 38 \\
\cline { 2 - 6 } & Non serous & $21(84.0)$ & $3(12.0)(p=0.37)$ & $1(4.0)(p=0.39)$ & 25 \\
\cline { 2 - 6 } & All & $54(85.71)$ & $7(11.11)(p=0.1)$ & $2(3.17)(p=0.73)$ & 63 \\
\hline \multirow{3}{*}{ Stage II } & Serous & $17(94.45)$ & $0(p=0.28)$ & $1(5.55)(p=0.79)$ & 18 \\
\cline { 2 - 7 } & Non serous & $13(100.0)$ & $0(p=0.37)$ & $0(p=0.39)$ & 13 \\
\cline { 2 - 7 } & All & $30(96.78)$ & $0(p=0.1)$ & $1(3.22)(p=0.79)$ & 31 \\
\hline \multirow{3}{*}{ Stage III } & Serous & $38(92.68)$ & $2(4.88)(p=0.28)$ & $1(2.44)(p=0.79)$ & 41 \\
\cline { 2 - 7 } & Non serous & $31(93.94)$ & $2(6.06)(p=0.37)$ & $0(p=0.39)$ & 33 \\
\cline { 2 - 7 } & All & $69(93.25)$ & $4(5.40)(p=0.1)$ & $1(1.35)(p=0.79)$ & 74 \\
\hline
\end{tabular}


one regular-sized recipient paraffin block containing 540 specimens. Five $\mu \mathrm{m}$ sections of the blocks were transferred to glass slides using a paraffin-sectioning aid system (adhesive coated slides, adhesive tape, UV-lamp; Instrumedics Inc., Hackensack, NJ, USA).

Fluorescence in situ Hybridization (FISH). Prior to hybridization the slides were treated with xylene at $37^{\circ} \mathrm{C}$, absolute ethanol at room temparature and $1 \mathrm{M}$ sodium thiocyanate at $80^{\circ} \mathrm{C}$ for deparrafinization and pretreatment. Dual color FISH was performed using the locus RP11-126K1 (1q21.3) clone probe, conjugated with digoxigenin and detected in red, and locus RP11-97G24 (1q23.3) clone probe, conjugated with biotin and detected in green. Labeling of the probes was performed by nick-translation. Denaturation of the DNA was carried out at $75^{\circ} \mathrm{C}$ for $10 \mathrm{~min}$. (probe mixture) or at $73^{\circ} \mathrm{C}$ for 5 min. (slides). The probe mixture was applied to the slides and hybridized overnight in a moist chamber at $37^{\circ} \mathrm{C}$. The post hybridization washes were performed using formamide $50 \% / 2 \times \mathrm{SSC}$. A threestep detection was achieved by blocking non specific signals with block buffer, first detection with FITC-avidin/ mouse anti-Dig, again blocking, second detection with anti-avidin/Dig anti-mouse IgG, blocking, and third detection with FITC-avidin/ anti-Dig rhodamine. The slides were counterstained with DAPI in antifade. The presence of more than five locus signals per cell or tight clusters in at least $10 \%$ of tumor cells was considered to connote amplification. The presence of 3-5 locus signals in at least $10 \%$ of tumor cells was considered to connote a gain.

Statistical Analysis. The relationship between copy number changes and clinicopathological data was estimated using the $\chi 2$ test and the $p$ value was calculated. A $p$ value of $<0.05$ was required for significance.

\section{RESULTS}

Of the 540 ovarian tumors we analyzed by FISH, we found signals in 289 tumors (53.5\%) for $1 \mathrm{q} 21.3$ and in 240 tumors (44.4\%) for 1q23.3. Copy number changes (both gain/amplification) were established in $9.64 \%$ of ovarian cancers regarding $1 \mathrm{q} 21.3$ and in $17.78 \%$ of ovarian cancers regarding $1 \mathrm{q} 23.3$.
Amplification of 1q21.3 and 1q23.3 and Degree of Malignancy. We found amplification of 1q21.3 as clusters in four malignant ovarian tumors $(2.41 \%)$ and none in LMP and benign ovarian tumors. Amplification of 1q23.3 was found as clusters in only two malignant tumors $(1.48 \%)$. The number of tumors with amplification was too small for statistical analysis.

Gain of 1q21.3 and 1q23.3 and Degree of Malignancy. We found between 3-5 signals for 1q21.3 (genetic gain, which could be due to partial or total trisomy 1$)$ in 11 malignant tumors $(6.63 \%)$, in two LMP tumors $(8.33 \%)$ and in 13 benign tumors $(13.13 \%)$. There was no statistically significant difference between the three groups when analyzing all tumors together. However, in the serous subtype 1q21.3 gain was statistically more frequent ( $p$ $<0.05$ ) in benign compared to LMP and malignant tumors (18.18 vs. 0.0 and 5.40\%). In the non serous tumors there was no significance.

We detected genetic gain of 1q23.3 in 22 malignant tumors $(16.30 \%)$, in three LMP tumors $(16.67 \%)$ and in 11 benign tumors (12.64\%). In the group of non epithelial tumors, we found significant increase in frequency of gain with the degree of malignancy (benign: $5.55 \%$, LMP: $0 \%$, malignant: $50 \%)(p<0.04)$.

Copy Number Changes of 1q21.3 and Histology. Genetic gain of 1q21.3 was found only in epithelial malignant ovarian tumors and not in the small number (seven) of non epithelial ovarian neoplasms. In epithelial carcinomas, there was a trend for significantly higher frequency in non serous carcinomas $(12.5 \%)$ than in serous carcinomas $(5.40 \%)$ $(p<0.12)$. The highest frequency was found in mucinous carcinomas (18.18\%), followed by undifferentiated carcinomas (16.67\%) (data not presented).

In epithelial LMP tumors, there was significantly more frequent genetic gain in non serous LMP tumors $(22.22 \%)$ than in serous LMP tumors $(0 \%)$ $(p<0.06)$. The highest frequency was found in mucinous LMP tumors $(25 \%)$.

The frequency of genetic gain did not differ significantly between epithelial (13.92\%) and non epithelial (10\%) benign ovarian tumors (Table 2). In epithelial benign ovarian tumors, there was no significant difference in frequency of genetic gain between serous adenomas (18.18\%) and non serous adenomas $(12.28 \%)$ (Table 2$)$. The highest fre- 
Table 2. Copy number changes of 1q21.3 in different grade tumors

\begin{tabular}{|c|c|c|c|c|c|}
\hline & & Normal Copy Number (\%) & Genetic Gain (\%) & Amplification (\%) & Total $(\%)$ \\
\hline \multirow[t]{4}{*}{ G1 } & Serous & $36(92.31)$ & $3(7.69)(p=0.47)$ & $0(p=0.41)$ & 39 \\
\hline & Non serous & $13(92.86)$ & $1(7.14)(p=0.15)$ & $0(p=0.3)$ & 14 \\
\hline & Non epithelial & 1 & 0 & 0 & 1 \\
\hline & All & $50(92.60)$ & $4(7.40)(p=0.54)$ & $0(p=0.03)$ & 54 \\
\hline \multirow[t]{4}{*}{ G2 } & Serous & $48(90.57)$ & $3(5.66)(p=0.47)$ & $2(3.77)(p=0.41)$ & 53 \\
\hline & Non serous & $14(93.33)$ & $0(p=0.15)$ & $1(6.67)(p=0.3)$ & 15 \\
\hline & Non epithelial & 3 & 0 & 0 & 3 \\
\hline & All & $65(91.56)$ & $3(4.22)(p=0.54)$ & $3(4.22)(p=0.03)$ & 71 \\
\hline \multirow[t]{4}{*}{ G3 } & Serous & $18(94.74)$ & $0(p=0.47)$ & $1(5.26)(p=0.41)$ & 19 \\
\hline & Non serous & $17(80.95)$ & $4(19.05)(p=0.15)$ & $0(p=0.3)$ & 21 \\
\hline & Non epithelial & 3 & 0 & 0 & 3 \\
\hline & All & $38(88.37)$ & $4(9.30)(p=0.54)$ & $1(2.32)(p=0.03)$ & 43 \\
\hline
\end{tabular}

quency was found in mucinous $(21.43 \%)$, followed by serous (18.18\%) and endometroid (11.43\%) adenomas (data not presented).

Amplification of 1q21.3 did not differ significantly in frequency between serous $(2.70 \%)$ and non serous $(2.08 \%)$ ovarian carcinomas. The frequency was highest in mucinous carcinomas $(9.09 \%)$.

Copy Number Changes of 1q23.3 and Histology of Ovarian Tumors. We found significantly higher frequency of genetic gain in 1q23.3 in non epithelial malignancies (50\%) compared to epithelial carcinomas $(14.73 \%)(p<0.03)$. There was no significant difference between serous $(12.5 \%)$ and non serous $(18.37 \%)$ carcinomas. The highest frequency was established in undifferentiated carcinomas $(41.18 \%)$. In LMP tumors, the frequency of the genetic gain was higher in non serous $(25 \%)$ than in serous tumors $(11.11 \%)$ but this did not reach statistical significance. In benign ovarian tumors, epithelial and non epithelial tumors did not differ significantly for 1q23.3 genetic gain (14.49 vs. 5.55\%). There was a trend towards higher frequency of the genetic gain in serous $(22.73 \%)$ than in non serous (10.64\%) adenomas.

Copy Number Changes of 1q21.3 and Tumor Phenotype. The frequency of genetic gain in 1q21.3 decreased from stage I to stages II-III (11.11 to 0.0 to $5.40 \%$ ) without reaching statistical significance for association with the early stage $(p=0.1$;
Table 1). There was no significant difference in the frequency of amplification in stage I, stage II and stage III $(3.17,3.22$ and $1.35 \% ; p 0.73)$ ovarian malignancies (Table 1). We did not find a statistically significant association with different stages in serous and non serous carcinomas (Table 1). Taken together, genetic gain and amplification of 1q21.3 showed a trend towards higher frequencies in stage I than in stage II and stage III (14.28 to 3.22 and $6.75 \%)(p<0.15)$.

We did not find an association with histological grades or for genetic gain $(\mathrm{G} 1: 7.40 \%, \mathrm{G} 2: 4.22 \%$, G3: $9.30 \% ; p 0.54)$ or for the amplification $(\mathrm{G} 1$ : $0 \%, \mathrm{G} 2: 4.22 \%, \mathrm{G} 3: 2.32 \% ; p 0.3$ ) (see Table 2), and similarly for combined copy number changes (genetic gain and amplification). If we took tumors from different grades separately in serous and non serous groups, the genetic gain of 1q21.3 showed a trend towards association with the degree of dedifferentiation (histological grade) in the non serous carcinomas (G1: 7.14\%, G2: 0\%, G3: 19.05\%) $(p=0.15)$ (Table 2).

Copy Number Changes of 1q23.3 and Tumor Phenotype. Overall, the genetic gain of 1q23.3 was not associated with tumor stages (stage I: $12.90 \%$, stage II: $10.53 \%$, stage III: $22.22 \% ; p=0.24$ ) (see Table 3). This did not change in the case of different stages of serous and non serous tumors.

We found an association of genetic gain of 1q23.3 
Table 3. Copy number changes of 1q23.3 in different stage tumors

\begin{tabular}{|l|l|c|c|c|c|}
\hline \multicolumn{2}{|c|}{} & Normal Copy Number (\%) & Genetic Gain (\%) & Amplification (\%) & Total (\%) \\
\hline \multirow{3}{*}{ Stage I } & Serous & $32(88.89)$ & $4(11.11)(p=0.26)$ & $0(p=0.24)$ & 36 \\
\cline { 2 - 6 } & Non serous & $20(90.91)$ & $2(9.09)(p=0.27)$ & $0(p=0.26)$ & 22 \\
\cline { 2 - 6 } & All & $54(87.10)$ & $8(12.90)(p=0.24)$ & $0(p=0.24)$ & 62 \\
\hline \multirow{3}{*}{ Stage II } & Serous & $11(91.67)$ & $0(p=0.26)$ & $1(p=0.24)$ & 12 \\
\cline { 2 - 6 } & Non serous & $4(66.67)$ & $2(33.33)(p=0.27)$ & $0(p=0.26)$ & 6 \\
\cline { 2 - 6 } & All & $16(84.21)$ & $2(10.53)(p=0.24)$ & $1(5.26)(p=0.24)$ & 19 \\
\hline \multirow{3}{*}{ Stage III } & Serous & $25(78.13)$ & $6(18.75)(p=0.26)$ & $1(3.12)(p=0.24)$ & 32 \\
\cline { 2 - 6 } & Non serous & $16(76.19)$ & $5(23.81)(p=0.27)$ & $0(p=0.26)$ & 21 \\
\cline { 2 - 6 } & All & $41(75.93)$ & $12(22.22)(p=0.24)$ & $1(1.85)(p=0.24)$ & 54 \\
\hline
\end{tabular}

${ }^{a}$ Also including non epithelial tumors.

with the degree of dedifferentiation (G1: $15.38 \%$, G2: $8 \%, \mathrm{G} 3: 26.07 \%)(p<0.05)$ (see Table 4). This association was also present for non serous carcinomas (G1: 9.09\%, G2: 0\%, G3: 29.63\%) $(p<0.06)$ and not for serous carcinomas (G1: $14.81 \%, \mathrm{G} 2$ : $8.11 \%, \mathrm{G} 3: 18.75 \% ; p=0.5$ ) (Table 4).

Combined Analysis of Copy Number Changes of 1q21.3 and 1q23.3. In all, 219 tumors were successfully analyzed for copy number changes in $1 \mathrm{q} 21.3$ and $1 \mathrm{q} 23.3$. We established genetic gains in 1q21.3 in 19 (eight malignant, one LMP and 10 be- nign tumors) and genetic gains in 1q23.3 in 32 (20 malignant, two LMP and 10 benign). Thus, genetic gain in 1q23.3 was 1.7-times more frequent than genetic gain in 1q21.3 in ovarian tumors. This difference was largest in malignant tumors $(20 v s$. 8). In benign ovarian tumors, there was no difference (10 tumors with gain for $1 \mathrm{q} 21.3$ and 10 with gain for 1q23.3).

Combined genetic gain in 1q21.3 and 1q23.3, which could be due to trisomy 1 , was found in 11 tumors (seven benign, one LMP and three malig-

Table 4. Copy number changes of 1q23.3 in different grade tumors

\begin{tabular}{|c|c|c|c|c|c|}
\hline & & Normal Copy Number (\%) & Genetic Gain (\%) & Amplification (\%) & Total $(\%)$ \\
\hline \multirow[t]{4}{*}{ G1 } & Serous & $23(85.19)$ & $4(14.81)(p=0.05)$ & 0 & 27 \\
\hline & Non serous & $10(90.91)$ & $1(9.09)(p=0.06)$ & 0 & 11 \\
\hline & Non epithelial & 0 & 1 & 0 & 1 \\
\hline & All & $33(84.62)$ & $6(15.38)(p=0.05)$ & 0 & 39 \\
\hline \multirow[t]{4}{*}{$\mathrm{G} 2$} & Serous & $32(86.49)$ & $3(8.11)(p=0.05)$ & $2(5.40)$ & 37 \\
\hline & Non serous & $11(100.0)$ & $0(p=0.06)$ & 0 & 11 \\
\hline & Non epithelial & 1 & 1 & 0 & 2 \\
\hline & All & $44(88.00)$ & $4(8.00)(p=0.05)$ & $2(4.00)$ & 50 \\
\hline \multirow[t]{4}{*}{ G3 } & Serous & $13(81.25)$ & $3(18.75)(p=0.05)$ & 0 & 16 \\
\hline & Non serous & $19(70.37)$ & $8(29.63)(p=0.06)$ & 0 & 27 \\
\hline & Non epithelial & 2 & 1 & 0 & 3 \\
\hline & All & $34(73.93)$ & $12(26.07)(p=0.05)$ & 0 & 46 \\
\hline
\end{tabular}


nant). Single genetic gain in 1q21.3 was established in eight tumors (three benign and five malignant) Tables 5 and 6). Single genetic gain for 1q23.3 was detected in 21 tumors (three benign, one LMP and 17 malignant) (Table 5 and 6).

The single genetic gain in 1q23.3 was significantly more frequent in ovarian tumors than that in $1 \mathrm{q} 21.3(p<0.015)$ (Table 4). This was especially evident in the malignant ovarian tumors with gains: three were with combined changes (12\%), five with single gain of $1 \mathrm{q} 21.3(20 \%)$ and 17 with single gain of 1q23.3 (68\%) $(p<0.007)$ (Table 6).

In contrast to malignant tumors, in benign ovarian tumors, combined copy number changes (referred to trisomy 1) prevailed (frequency of $53.85 \%$ for all benign tumors with gains), and the frequency of single gains of 1q21.3 and 1q23.3 were similar (each of $23.08 \%$ ) (Table 6). The single gain in $1 \mathrm{q} 23.3$ occurred only in three endometroid cysts and single gain in 1q21.3 in two endometroid and one mucinous adenoma.

\section{DISCUSSION}

This is the first study of copy number changes for specific BAC clones in 1q21.3 and 1q23.3 in ovarian tumors. Such changes in 1q21.3 were established in $9.64 \%$ of malignant (2.41\% amplification), in $8.33 \%$ of LMP and in $13.13 \%$ of benign ovarian tumors. Copy number changes in 1q23.3 were found in $17.78 \%$ of malignant (1.48\% amplification), in $16.67 \%$ of LMP and in $12.64 \%$ of benign ovarian tumors.
Gain in 1q21-q23 has been reported in other types of tumors, with a frequency of $2-30 \%$ for hepatic cancers $[13,14]$, of $10 \%$ for head and neck cancers $[15,16]$, of $4-9 \%$ for non small $[17,18]$ and of $5 \%$ for small lung cancers [19] and of $2-8 \%$ for bladder cancers $[20,21]$. We found the frequency of these alterations for ovarian cancers to be $9.64 \%$ for $1 \mathrm{q} 21.3$ and $17.78 \%$ for $1 \mathrm{q} 23.3$. Interestingly, gain of 1q32-q43 occurred in $30-58 \%$ of ovarian cancers according to other authors $[22,23]$. We suggest that genetic alterations in chromosome 1q in ovarian tumors increases with distance from the centromere and are more frequent in the distal part of 1q.

We found that benign ovarian tumors were affected mainly by trisomy 1 , whereas malignant ovarian tumors were affected by more complex rearrangements, in particular in 1q21.3 and 1q23.3. A very small number of benign tumors, particularly endometroid ones, contained such changes, probably because these are pre cancerous lesions. Genetic gain in 1q23.3 was 2.5-times more frequent in ovarian cancers than in gain in 1q21.3. Single gain in 1q23.3 was significantly more frequent in malignant ovarian tumors, possessing gains in the analyzed regions.

Using CGH in primary ovarian cancers, 1q gain was shown in $32 \%$ of the cases [23] and in $38 \%$ of ovarian cancers [24]. In the latter, 1q22-q32 amplification was found in $29 \%$ of tumors. Taking into account the low frequency of amplification in 1q23.3 that we found, the difference is likely due to the amplification of 1q32.

Table 5. Combined analysis of 1q21.3 and 1q23.3 gain in ovarian tumors

\begin{tabular}{|l|c|c|}
\hline & Normal Copy Number 1q23.3 & Genetic Gain 1q23.3 \\
\hline Normal copy number 1q21.3 & $177(81.74 \%)$ & $21(9.59 \%)$ \\
\hline Genetic gain 1q21.3 & $8(3.65 \%)$ & $11(5.02 \%)$ \\
\hline
\end{tabular}

Table 6. Distribution of combined and independent gains of 1q21.3 and 1q23.3 in benign, low malignant potential and malignant tumors

\begin{tabular}{|l|c|c|c|c|}
\hline Normal Gained & $\begin{array}{c}\text { Normal 1q23.3+ } \\
\text { Gain 1q21.3 }\end{array}$ & $\begin{array}{c}\text { Gain 1q23.3+ } \\
\text { Gain 1q21.3 }\end{array}$ & $\begin{array}{c}\text { Gain 1q23.3+ } \\
\text { Normal 1q21.3 }\end{array}$ & Total \\
\hline Benign & $3(23.08 \%)$ & $7(53.85 \%)$ & $3(23.08 \%)$ & 13 \\
\hline LMP & 0 & $1(50.0 \%)$ & $1(50.0 \%)$ & 2 \\
\hline Malignant & $5(20.0 \%)$ & $3(12.0 \%)$ & $17(68.0 \%)$ & 25 \\
\hline
\end{tabular}


We found significantly more frequent gain in $1 \mathrm{q} 23.3$ in non epithelial tumors $(50 \%)$ compared to epithelial tumors $(14.73 \%)(p<0.03)$. Similarly, 1q gain was considered as the most frequent in germline cell tumors in both sexes, especially in childhood $[25,26]$. Gain of $1 \mathrm{q}$ was found in six out of $21(28.5 \%)$ malignant germline cell tumors [27] and in three out of four germline cell tumors [28]. In our study gain in $1 \mathrm{q} 21.3$ was not established in non epithelial tumors. Our results assumed that gains in 1q23.3 are characteristic of non epithelial tumors, but not gains in $1 \mathrm{q} 21.3$.

We found the highest frequency for $1 \mathrm{q} 21.3$ in mucinous cancers $(41.18 \%)$ and for $1 \mathrm{q} 23.3$ in undifferentiated cancers (18.18\%). The gain of 1q21.3 prevailed in non serous malignant and LMP ovarian tumors in comparison to serous tumors. Another study found that $1 \mathrm{q}$ gain associated with the serous type and this is probably referred to the distal region of $1 \mathrm{q}$.

Of particular importance for the clinical course of the disease is the stage and the histological grade of a tumor. In different stage tumors, we found no statistical significance for the gain in 1q21.3, though its frequency decreased from stage I to stages II-III. No association with tumor stage was established for copy number changes in 1q23.3. In tumors with other localizations, such associations were also not found, alterations of chromosome 1 being considered to be non specific [29] except for neuroblastomas, in which gain in 1q23 was closely related to disease progression and aggressiveness [30].

Copy number changes in 1q21.3 were not associated with histological grade for all tumors taken together, but in non serous tumors there was a trend for association with the higher grade. This association for 1q23.3 showed significantly higher frequency of its gain in higher grade tumors, especially in non serous tumors.

Many investigators considered alterations of chromosome 1 to be non specific. This is probably due to the fact that chromosome 1 contains a large amount of about 2,500 genes and that different regions/genes are altered in different tumors. Thus, information is needed to confirm non specificity. It is necessary to investigate all the loci of chromosome 1 in a large number of tumors in order to determine the most likely candidate genes for ovarian carcinogenesis.

\section{REFERENCES}

1. Whang-Peng J, Knutsen T, Douglass EC, Chu E, Ozols RF, Hogan WM, Young RC. Cytogenetic studies in ovarian cancer. Cancer Genet $\mathrm{Cy}-$ togenet 1984; 11(1): 91-106.

2. Woods LK, Morgan RT, Quinn LA, Moore GE, Semple TU, Stedman KE. Comparison of four new cell lines from patients with adenocarcinoma of the ovary. Cancer Res 1979; 39(11): 44494459.

3. Atkin N, Baker M. Specific chromosome change in ovarian cancer. Cancer Genet Cytogenet 1981; 3(3): 275-276.

4. Bayani J, Brenton JD, Macgregor PF, Beheshti B, Albert M, Nallainathan D, Karaskova J, Rosen B, Murphy J, Laframboise S, Zanke B, Squire JA. Parallel analysis of sporadic primary ovarian carcinomas by spectral karyotyping, comparative genomic hybridization, and expression microarrays. Cancer Res 2002; 62(12): 3466-3476.

5. Taetle R, Aickin M, Panda L, Emerson J, Roe D, Thompson F, Davis J, Trent J, Alberts D. Chromosome abnormalities in ovarian adenocarcinoma: II. Prognostic impact of nonrandom chromosome abnormalities in 244 cases. Genes Chromosomes Cancer 1999; 25(1): 46-52.

6. Kallioniemi A, Kallioniemi O, Sudar D, Rutovitz D, Gray JW, Waldman F, Pinkel D. Comparative genomic hybridization for molecular cytogenetic analysis of solid tumors. Science 1992; 258(5083): 818-821.

7. Sonoda G, Palazzo J, du Manoir S, Godwin AK, Feder M, Yakushiji M, Testa JR. Comparative genomic hybridization detects frequent overexpression of chromosomal material from 3q26, 8q24, and 20q13 in human ovarian carcinomas. Genes Chromosomes Cancer 1997; 20(4): 320-328.

8. Osterberg L, Levan K, Partheen K, Helou K, Horvath G. Cytogenetic analysis of carboplatin resistance in early-stage epithelial ovarian carcinoma. Cancer Genet Cytogenet 2005; 163(2): 144150.

9. Takano M, Fujii K, Kita T, Kikuchi Y, Uchida K. Amplicon profiling reveals cytoplasmic overexpression of MUC1 protein as an indicator of resistance to platinum-based chemotherapy in patients with ovarian cancer. Oncol Rep 2004; 12(6): 1177-1182. 
10. Helou K, Padilla-Nash H, Wangsa D, Karlsson E, Osterberg L, Karlsson P, Ried T, Knutsen T. Comparative genome hybridization reveals specific genomic imbalances during the genesis from benign through borderline to malignant ovarian tumors. Cancer Genet Cytogenet 2006; 170(1): $1-8$.

11. Kurman RJ, Ed. Blaustein's Pathology of the Female Genital Tract, 4th Ed. New York: Springer-Verlag. 1994: 705-939.

12. Kononen J, Bubendorf L, Kallioniemi A, Bärlund M, Schraml P, Leighton S, Torhorst J, Mihatsch MJ, Sauter G, Kallioniemi OP. Tissue microarray for high-throughput molecular profiling of hundreds of specimens. Nat Med 1998; 4(7): 844-847.

13. Wong N, Lai P, Lee SW, Fan S, Pang E, Liew CT, Sheng Z, Lau JW, Johnson PJ. Assessment of genetic changes in hepatocellular carcinoma by comparative genomic hybridization analysis: relationship to disease stage, tumor size, and cirrhosis. Am J Pathol 1999, 154(1): 37-43.

14. Kusano N, Shiraishi K, Kubo K, Oga A, Okita K, Sasaki K. Genetic aberrations detected by comparative genomic hybridization in hepatocellular carcinomas: their relationship to clinicopathological features. Hepatology 1999; 29(6): 1858-1862.

15. Bockmühl U, Schwendel A, Dietel $M$, Petersen I. Distinct patterns of chromosomal alterations in high- and low-grade head and neck squamous cell carcinoma. Cancer Res 1996; 56(23): 5325-5329.

16. Bockmühl U, Wolf G, Schmidt S, Schwendel A, Jahnke V, Dietel M, Petersen I. Genomic alterations associated with malignancy in head and neck cancer. Head Neck 1998; 20(2): 145-151.

17. Michelland S, Gazzeri S, Brambilla E, Robert-Nicoud M. Comparison of chromosomal imbalances in neuroendocrine and non-small-cell lung carcinomas. Cancer Genet Cytogenet 1999; 114(1): 22-30.

18. Petersen I, Bujard M, Petersen S, Wolf G, Goeze A, Schwendel A, Langreck H, Gellert K, Reichel M, Just K, du Manoir S, Cremer T, Dietel M, Ried T. Patterns of chromosomal imbalances in adenocarcinoma and squamous cell carcinoma of the lung. Cancer Res 1997; 57(12): 2331-2335.

19. Petersen I, Langreck H, Wolf G, Schwendel
A, Psille R, Vogt P, Reichel MB, Ried T, Dietel M. Small-cell lung cancer is characterized by a high incidence of deletions on chromosomes 3p, 4q, 5q, 10q, 13q and 17p. Br J Cancer 1997; 75(1): 79-86.

20. Richter J, Jiang F, Görög J-P, Sartorius G, Egenter C, Gasser TC, Moch H, Mihatsch MJ, Sauter G. Marked genetic differences between stage pTa and stage pT1 papillary bladder cancer detected by comparative genomic hybridization. Cancer Res 1997; 57(14): 2860-2864.

21. El-Rifai W, Kamel D, Larramendy ML, Shoman S, Gad Y, Baithun S, El-Awady M, Eissa S, Khaled H, Soloneski S, Sheaff M, Knuutila S: DNA copy number changes in schistosoma-associated and non-schistosoma-associated bladder cancer. Am J Pathol 2000; 156(3): 871-887.

22. Hauptmann S, Denkert C, Koch I, Petersen S, Schlüns K, Reles A, Dietel M, Petersen I. Genetic alterations in epithelial ovarian tumors analyzed by comparative genomic hybridization. Hum Pathol 2002; 33(6): 632-641.

23. Sham JST, Tang TC-M, Fang Y, Sun L, Qin L-X, Wu Q-L, Xie D, Guan X-Y. Recurrent chromosome alterations in primary ovarian carcinoma in Chinese women. Cancer Genet Cytogenet 2002; 133(1): 39-44.

24. Tapper J, Butzow R, Wahlstrom T, Seppala M, Knuutila S. Evidence for divergence of DNA copy number changes in serous, mucinous and endometrioid ovarian carcinomas. Br J Cancer 1997; 75(12): 1782-1787.

25. Schneider D, Schuster AE, Fritsch MK, Calaminus G, Harms D, Göbel U, Perlman EJ. Genetic analysis of childhood germ cell tumors with comparative genomic hybridization. Klin Padiatr 2001; 213(4): 204-211.

26. Bussey KJ, Lawce HJ, Olson SB, Arthur DC, Kabusek DK, Krailo M, Giller R, Heifetz S, Womer R, Magenis RE. Chromosome abnormalities of eighty-one pediatric germ cell tumors: sex-, age-, site-, and histo-pathology-related differences--a Children's Cancer Group study. Genes Chromosomes Cancer 1999; 25(2): 134-146.

27. Riopel MA, Spellerberg A, Groffin CA, Perlman EJ. Genetic analysis of ovarian germ cell tumors by comparative genomic hybridization. Cancer Res 1998; 58(14): 3105-3110.

28. Kraggerud SM, Szymanska J, Abeler VM, Kaern J, Eknaes M, Heim S, Teixeira MR, Trope 
CG, Peltomaki P, Lothe RA. DNA copy number changes in malignant ovarian germ cell tumors. Cancer Res 2000; 60(11): 3025-3030.

29. Thompson F, Liu Y, Emerson J, Weinstein R, Makar R, Trent J, Taetle J, Alberts D. Simple numeric abnormalities as primary karyotype changes in ovarian carcinoma. Genes Chromosomes Can- cer 1994; 10(4): 262-266.

30. Hirai M, Yoshida S, Kashiwagi T, Kawamura T, Ishikawa T, Kaneko M, Ohkawa H, Nakagawara A, Miwa M, Uchida K. 1q23 gain is associated with progressive neuroblastoma resistant to aggressive treatment. Genes Chromosomes Cancer 1999; 25(3): 261-269. 